\title{
Mild Traumatic Brain Injury and Treatment Response in Prolonged Exposure for PTSD
}

\author{
Rebecca K. Sripada, ${ }^{1}$ Sheila A. M. Rauch, ${ }^{1}$ Peter W. Tuerk, ${ }^{2}$ Erin Smith, ${ }^{1}$ Andrew M. Defever, ${ }^{1}$ \\ Rebecca A. Mayer, ${ }^{1}$ Michael Messina, ${ }^{1}$ and Margaret Venners ${ }^{1}$ \\ ${ }^{1}$ VA Ann Arbor Health Care System/University of Michigan Medical School, Ann Arbor, Michigan, USA \\ ${ }^{2}$ Ralph H. Johnson VAMC/Medical University of South Carolina, Charleston, South Carolina, USA
}

\begin{abstract}
A proportion of U.S. veterans returning from Iraq and Afghanistan have experienced mild traumatic brain injury (mTBI), which is associated with increased risk for developing posttraumatic stress disorder (PTSD). Prolonged Exposure (PE) has proven effectiveness in the treatment of PTSD; however, some clinicians have reservations about using PE with individuals with a history of mTBI. We examined the impact of PE for veterans with PTSD and with or without a history of mTBI in a naturalistic sample of 51 veterans who received PE at a Veterans Health Administration PTSD clinic. We also analyzed previously collected data from a controlled trial of 22 veterans randomly assigned to PE or present centered therapy. For both sets of data, we found that PE reduced symptom levels and we also did not detect an effect for mTBI, suggesting that PE may be helpful for individuals with PTSD and a history of mTBI.
\end{abstract}

Over two million individuals (Defense Manpower Data Center, 2009) have been deployed to Iraq or Afghanistan as part of Operation Enduring Freedom (OEF), Operation Iraqi Freedom (OIF), and/or Operation New Dawn (OND). A small proportion (between 10-20\%; Hoge et al., 2008; Polusny et al., 2011) experience mild traumatic brain injury (mTBI). Specifically, mTBI is (American Congress of Rehabilitation Medicine, 1993)

\begin{abstract}
A traumatically induced physiological disruption of brain function, as manifested by at least one of the following: 1. any period of loss of consciousness [not exceeding 30 minutes]; 2 . any loss of memory for events immediately before or after the accident; 3 . any alteration in mental state at the time of the accident (e.g., feeling dazed, disoriented, or confused); and 4. focal neurological deficit(s) that may or may not be transient [such as weakness, loss of balance, and vision changes]. (p. 1)
\end{abstract}

In most cases, symptoms associated with mTBI resolve within weeks or months; however, 7-33\% of individuals who experience mTBI report symptoms that persist past 3-months postinjury (Belanger, Curtiss, Demery, Lebowitz, \& Vanderploeg, 2005). These symptoms typically include headache,

Correspondence concerning this article should be addressed to Rebecca K. Sripada, Ph.D. VA Serious Mental Illness Treatment Resource and Evaluation Center (SMITREC) Ann Arbor, MI. E-mail: rekaufma@umich.edu

Published 2013. This article is a US Government work and is in the public domain in the USA. View this article online at wileyonlinelibrary.com DOI: $10.1002 /$ jts. 21813 dizziness, memory problems, balance problems, and irritability, and are collectively referred to as prolonged postconcussive syndrome (PPCS; Brenner et al., 2010).

Rates of head injury in OEF/OIF are 16-21\% higher than those in World War II, Korea, and Vietnam wars (Owens et al., 2008). This may be partially due to a rise in the use of improvised explosive devices, "devices placed or fabricated in an improvised manner incorporating destructive, lethal, noxious, pyrotechnic or incendiary chemicals, designed to destroy, disfigure, distract or harass ..." (pg. GL3) (U.S. Department of Defense [DoD], 1998). Other likely contributors to high rates of head injury are body armor improvements and rapidity of medical evacuations, which improve the rate of survival after severe injury (Owens et al., 2008). Multiple head injuries and/or loss of consciousness increase the likelihood of developing both posttraumatic stress disorder (PTSD) and PPCS (Schneiderman, Braver, \& Kang, 2008). mTBI has been named the "signature injury" of OEF/OIF/OND (Independent Review Group, 2007), and the U. S. Department of Veterans Affairs (VA) and DoD have allocated significant resources toward examining the potential impact of mTBI on the treatment of co-occurring conditions.

Although most veterans do not experience long-term mental health issues related to deployment, approximately $20 \%$ do report problems, including PTSD, depression, and/or alcohol and substance misuse (Tanielian \& Rand Corp., 2008). PTSD is an anxiety disorder characterized by re-experiencing, avoidance, and hyperarousal symptoms that develop after exposure to a traumatic event (American Psychiatric Association 
[APA], 2000). PTSD is diagnosed in $10-20 \%$ of returning veterans (Polusny et al., 2011); however, for those with mTBI, rates of PTSD may be as high as 40\% (Carlson et al., 2009; Polusny et al., 2011). Controversy pervades discussion of these co-occurring conditions, with disagreement regarding whether they are independent problems or manifestations of a common substrate (Hoge, Goldberg, \& Castro, 2009). This controversy stems from significant overlap in the symptom profile and health outcomes of PTSD and mTBI (Brenner et al., 2010; Polusny et al., 2011). Both are associated with fatigue, irritability, poor sleep, and impaired concentration, attention, and memory. Data from several studies, however, demonstrate that postconcussive symptoms and psychosocial outcomes associated with mTBI are largely attributable to PTSD symptoms rather than mTBI itself (Hoge et al., 2008; Polusny et al., 2011; Schneiderman et al., 2008). Regardless of the diathesis of these issues, the fact that interventions shown to reduce PTSD symptoms are available suggest that such treatment is a reasonable and valid point of initial treatment for people who experienced mTBI and continue to suffer symptoms of PTSD.

Cognitive-behavioral therapy (CBT) has emerged as a gold standard treatment for PTSD, with extensive research supporting its efficacy (Bisson et al., 2007; Rauch, Eftekhari, \& Ruzek, 2012). Prolonged exposure (PE; a form of CBT) shows efficacy with both civilians (Powers, Halpern, Ferenschak, Gillihan, \& Foa, 2010) and veterans (Rauch et al., 2009; Schnurr et al., 2007; Tuerk et al., 2011). Despite its proven efficacy for the treatment of PTSD, some clinicians have concerns about using PE for PTSD in patients with a history of mTBI. Hesitance to use PE may be due to anecdotal concerns that loss of consciousness during a TBI will interfere with memory encoding, and thus preclude re-experiencing symptoms (Bryant, 2001; Joseph \& Masterson, 1999) or render treatments that rely on narrative accounts of the trauma (such as PE) less effective (Verfaellie, Amick, \& Vasterling, 2012). This hesitancy may also be due to a reasonable, but underexplored belief that cognitive impairment from TBI might reduce the efficacy of CBT (Elder, Mitsis, Ahlers, \& Cristian, 2010; Otis, McGlinchey, Vasterling, \& Kerns, 2011). Some have also voiced concerns that chronic pain, deficits in emotion regulation, or reduced impulse control could reduce the effectiveness or limit engagement in trauma-focused treatment by PTSD patients with a history of mTBI (Bryant \& Hopwood, 2006). Recent studies have begun to investigate the efficacy of PE and cognitive processing therapy (CPT; another form of CBT) for individuals with a history of mTBI (Chard, Schumm, McIlvain, Bailey, \& Parkinson, 2011; Walter, Kiefer, \& Chard, 2012; Wolf, Strom, Kehle, \& Eftekhari, 2012). No controlled studies of treatment for PTSD in mTBI, however, have been published. This represents a critical gap given the importance of providing efficacious treatment to individuals with PTSD who experienced mTBI.

In this study, we assessed the impact of PE for individuals with and without a history of TBI. We examined the treatment correlates of TBI in two different studies. In Study 1, we ex- amined the clinical impact of PE through a chart review of a clinical sample of 51 veterans who received PTSD treatment in a Veterans Health Administration (VHA) PTSD clinic. In Study 2 , we examined secondary data from a randomized controlled trial of 22 VA patients randomly assigned to PE or present centered therapy (PCT). Given the extensive overlap in symptomatology and outcomes between PTSD and mTBI, as well as the simplicity, flexibility, and individualized nature of PE, we expected that PE would be helpful for individuals with PTSD, and that the presence of mTBI would not impact the effect of PE.

\section{Study 1}

\section{Method}

Participants and procedure. For Study 1, all patients were diagnosed with PTSD and treated with PE in a VHA PTSD clinic as part of routine clinical care in an urban VA Medical Center (VAMC). No protocol-related exclusion criteria or incentives to participate in treatment were in place. These data were collected with approval from the institutional review board (IRB) for the VA Ann Arbor Healthcare System.

The sample consists of all veterans who began PE treatment between October 24, 2005 and June 7, 2011 and completed the PTSD Symptom Checklist in accordance with the therapy protocol, with veterans from Vietnam, OEF/OIF/OND, and other eras represented. Patients were identified for treatment through referrals from general mental health providers, case managers, and primary care providers to the VHA PTSD clinic. Before beginning PE, patients received comprehensive psychiatric assessment with the Mini-International Neuropsychiatric Interview (MINI; Sheehan et al., 1998), and were formally assessed for a PTSD diagnosis with the Clinician Administered PTSD Scale (CAPS; Blake, Weathers, Nagy, \& Kaloupek, 1995). Clinical interviews were performed by experienced mastersor doctoral-level clinicians with extensive training in the CAPS and MINI. Eleven patients (22\%) were positive for a CPRSdefined TBI. Nine of these 11 patients were classified as positive for TBI based on TBI consultation or service connection, and two were classified via problem list. Mean age was 49.3 years, and 59\% of patients were married. Four percent of patients were Black and $96 \%$ were White. Approximately 50\% had other medical conditions, $17 \%$ had comorbid substance use disorders, and $64 \%$ comorbid psychiatric diagnoses. Thirtyseven percent served in Vietnam, 33\% in the Persian Gulf, 4\% in Afghanistan, and 28\% in Iraq.

Therapist assignment was based on provider availability as part of normal operating procedures of the outpatient specialty PTSD clinic. Treatment occurred in standard clinic practice. As such, treatment sessions were not coded for treatment fidelity. All treatment providers were licensed social workers or psychologists practicing in a specialized PTSD clinic, or clinical social work or psychology trainees working under close 
supervision with PTSD clinic providers. All had completed specialized training in PE either through the VA rollout or through training from the senior author (S.A.M.R.).

Prolonged exposure (Foa, Hembree, \& Rothbaum, 2007) consists of four major components: (a) psychoeducation regarding common reactions to trauma, rationale for treatment and self-assessment; (b) repeated in vivo exposure to situations avoided due to trauma-related distress; (c) repeated, prolonged imaginal exposure to traumatic memories; and (d) emotional processing of the exposures. PE is usually delivered in 8-15 weekly, 90-minute sessions. In the current study, the length of treatment varied by patient and was informed by ongoing collaborative assessment within the context of normative clinical procedures. Given the individualized nature of the standard PE protocol, no modifications were made for individuals with a history of mTBI.

Measures. The PTSD Checklist-Specific Version (PCLS; Weathers, Huska, \& Keane, 1991) is a 17-item self-report measure of PTSD symptoms based on DSM-IV criteria (APA, 2000). Scores on the PCL-S range from 17 to 85 with higher scores reflecting greater PTSD severity. A score of 50 or above indicates clinical PTSD, and a change of 10 points or more is considered clinically significant (Monson et al., 2008). The instrument has good diagnostic efficiency $(>.70)$ and high internal consistency $(\alpha=.94$; Blanchard, Jones-Alexander, Buckley, $\&$ Forneris, 1996). Cronbach's $\alpha$ for this sample was .92.

In the current study, TBI was defined by the presence of TBI designation in the computerized patient record system (CPRS). Patients were assigned a value between 0 and 2 based on whether any of the following were present: (a) screened positive for TBI in CPRS and received follow-up formal TBI consultation at dedicated TBI clinics, (b) service connected for $\mathrm{TBI} /$ postconcussive syndrome, or (c) current CPRS problem list includes TBI-related issues (headaches that are connected to TBI in notes, cognitive impairment connected to TBI in notes, etc.). If all were negative then the score was zero. If any one of the three criteria was positive then the score was 1 . If evidence in CPRS indicated clearly more than mild TBI (i.e., penetrating gunshot wound, etc.) then a score of 2 was documented; however, preliminary investigation indicated no overt differences in PTSD symptoms or responses between those who scored 1 and those who scored 2. Accordingly, the levels were combined due to a low number of veterans scoring 2 . The result was a binary measure $(0,1)$ indicating the presence or absence of data documenting a potential TBI.

Data analyses. Repeated measures, dependent means $t$ tests were used to compare pre- and posttreatment effects on the PCL for the entire intent-to-treat (ITT) sample and for the treatment completers subsample. Statistically significant differences were qualified using $d$-type effect sizes. In the current study, treatment completers were defined as any patient attending at least eight sessions of $\mathrm{PE}$, with the exception that early termination (i.e., prior to Session 8) was not operationalized as attrition if the patient had achieved at least a 50\% reduction in PCL from baseline. In addition to analyzing pre- and posttreatment differences with $t$ tests, hierarchical linear modeling (HLM) was used to analyze the intention-to-treat (ITT) longitudinal data and to investigate the potential effect of mTBI status on treatment outcomes and the slope of outcomes. HLM is a practical strategy for analyzing effectiveness data because the method does not assume fixed time points of measurement or equal numbers of observations and missing data do not cause special problems (Raudenbush \& Bryk, 2002). Specifically, in the current study, HLM was used to investigate longitudinal PCL outcomes (Level 1) nested within patients (Level 2) to assess overall treatment response and response over time. Variance components sigma squared $\left(\sigma^{2}\right)$ and tao $(\tau)$ were estimated and used to calculate intraclass correlations. Statistically significant outcomes were qualified using between- and within- $R^{2}$-type effect sizes in a manner consistent with recommended guidelines (Snijders \& Bosker, 1994). HLM analyses were conducted using HLM software (Raudenbush, Bryk, Cheong, \& Congdon, 2004), and all other analyses were conducted using SPSS Statistics 19 (IBM Corporation, Armonk, NY).

\section{Results}

The average number of sessions for the total ITT sample was $10(S D=3.8)$ with 40 veterans $(78 \%)$ meeting criteria as treatment completers. The average number of sessions for treatment completers was $12(S D=2.7)$. Eleven veterans $(22 \%)$ did not complete at least eight sessions and were classified as noncompleters; these patients were retained for analysis in the ITT sample. Neither baseline PCL severity score nor TBI status were predictors of treatment completion or number of sessions.

Baseline PCL-S scores were consistent with previous studies with veteran samples (Schnurr et al., 2007; Tuerk et al., 2011). Mean pre- and posttreatment PCL-S scores for the ITT sample were $60(S D=13$, range $=29-85)$ and $47(S D=17)$. This difference is clinically and statistically significant, $t(49)=6.59$, $p<.001, d=1.00$. Mean pre- and posttreatment PCL-S scores for the treatment completer sample were $60.4(S D=13.4)$ and $45.3(S D=16.5)$. This difference is also clinically and statistically significant, $t(39)=7.19, p<.001, d=1.13$.

Considering the subsample of only those patients with mTBI, mean pre- and posttreatment PCL-S scores for the ITT sample were $58.9(S D=8.6)$ and $43.5(S D=14.1)$. This difference is clinically and statistically significant, $t(10)=3.65, p<.005$, $d=1.81$. Mean pre- and posttreatment PCL-S scores for the treatment completer sample were $61.0(S D=7.7)$ and 44.0 $(S D=14.3)$. This difference is also clinically and statistically significant, $t(7)=3.68, p<.01, d=2.22$.

Models of longitudinal PCL outcomes were based on 234 points of measurement (Level 1) nested within 51 patients (Level 2). The unconditional HLM model estimated variance components for Level-1 and Level-2 units $\left(\sigma^{2}=95.41\right.$, $\tau=152.85$ ). The value of $\tau$ was significantly different from zero, $\chi^{2}(50)=358.76, p<.001$, indicating the presence of 
patient-level effects on outcomes. The intraclass correlation (ICC) was .62, indicating that $62 \%$ of total PCL variance could be accounted for by factors associated with the patient ( $R^{2}$-between). Such factors could be related to personal characteristics (i.e., age or baseline pathology) or external factors that varied by patient (i.e., assigned therapist or seasonal effects). The remaining variance (38\%) could be attributed to withinpatient effects such as time in treatment or to other factors not included in the model.

The next sequential step in modeling involved adding weeks in treatment (i.e., occurrence of longitudinal measurement, or "session dose") as a Level-1 within-patient predictor. Weeks in treatment significantly predicted PCL outcomes, $\sigma^{2}=60.71, \tau$ $=165.45, \chi^{2}(50)=563.85, p<.001$. The modeled coefficient indicated linear decreases in PCL symptoms over time, $t(232)$ $=10.24, p<.001$. The weeks in treatment effect accounted for $9 \%$ of the variance in outcomes. Baseline PCL scores were not a significant predictor of treatment outcomes or the slope of outcomes.

With overall treatment effects accounted for, the next step in the model involved adding TBI status to explore potential disparities in outcomes. TBI status did not significantly predict PCL scores, $t(49)=-0.94, p=.35$, or the slope of scores over time, $t(49)=-0.39, p=.70$. Additionally, there were no statistically significant differences in time spent in treatment between groups, $t(48)=-0.84, p=.31$. Patients with and without a history of TBI spent an average of $6.5(S D=4.0)$ weeks and $5.5(S D=2.3)$ weeks in treatment, respectively. Accordingly, TBI status was omitted from the final parsimonious model (Table 1).

\section{Study 2}

\section{Method}

Participants and procedures. The following analyses were conducted as a post-hoc examination of data collected in a translational treatment mechanisms study. As such, the sample size is small and findings are preliminary. Due to small sample size and the design of the primary study, only treatment completers are included in the analyses. Treatment completion was defined as finishing 10-12 sessions per protocol and completing the posttreatment assessment. (For a full description of study procedures and the sample, see Rauch et al., 2013.) All veterans participated in the treatment study between January 2008 and July $2010(N=22 ; 9 \%$ female, 23\% African American, $73 \%$ White, 5\% Asian). Mean age was 32.7 years $(S D=6.9)$. With regard to combat location, $81 \%$ served in Iraq and 33\% served in Afghanistan, with some veterans reporting deployments to both locations. The same definition for TBI status was used as Study 1. Based on this definition, 36\% $(n=8)$ of the sample had experienced TBI (most mild, but some with more severe injuries including a penetrating head wound). No veterans were excluded due to cognitive impairment. As reported in the primary outcome article, comorbidity was representative of the OEF/OIF/OND population: $57 \%$ had depression or dysthymia, $10 \%$ had alcohol abuse, and $29 \%$ met criteria for another anxiety disorder at intake. All treatment was conducted by an experienced PE provider (S.A.M.R.) with over 12 years of experience using the protocol. All participants provided written informed consent, and all procedures were conducted with approval from the IRB for the VA Ann Arbor Healthcare System.

All veterans were randomly assigned to receive between 10 to 12 sessions of PE or PCT (Schnurr et al., 2007) based on clinical presentation at session 10. PCT uses a present-centered and problem-solving oriented approach to facilitate adaptive responses to ongoing stress and difficulties and assist the veteran in obtaining his or her highest level of functioning.

Measure. The Clinician Administered PTSD Scale (CAPS; Blake et al., 1995) is an interview measure of PTSD severity with excellent psychometric properties, including high internal consistency ( $\alpha=.94$; Blake et al., 1995). Cronbach's $\alpha$ for this sample was .95. Each of the 17 DSM-IV PTSD symptoms is rated on both frequency and intensity according to a 5-point (0-4) scale. These ratings are summed to compute a severity score for each symptom, and the symptom scores are summed to obtain an overall measure of PTSD severity.

Data analyses. We used repeated measures (pre-, mid-, post-) analysis of variance (ANOVA) to compare the impact of TBI (TBI vs. no TBI) on treatment effects for PTSD (CAPS). To provide additional information regarding the impact of TBI on treatment, planned follow-up repeated measures ANOVAs among TBI positive veterans were also conducted to compare the impact of treatment condition (PE vs. PCT) on CAPS. Given the small sample size, planned comparisons that approached significance and statistically significant differences were qualified using $d$-type effect sizes. HLM was not deemed necessary because this study employed fixed time points and did not have missing data.

\section{Results}

As reported elsewhere (Rauch et al., 2013), CAPS scores in the $\mathrm{PE}$ and PCT groups combined were significantly reduced over time, $F(2,40)=35.3, p<.001$, pre- $(M=78.4, S D=11.3)$, posttreatment $(M=41.4, S D=26.0)$, pre- to posttreatment $d=$ 1.85. Neither the main effect of TBI status, $F(1,20)=1.0$, not significant $(n s)$, nor the interaction between time and TBI, $F(2$, $40)=0.1, n s$-approached significance. Moreover, the mean pre- and post-CAPS scores between groups were very similar (no-TBI pre-CAPS $M=76.1, S D=10.8$ and post-CAPS $M$ $=39.1, S D=22.5$; TBI pre-CAPS $M=82.4, S D=11.7$ and post-CAPS $M=45.5, S D=32.5$ ).

Among the TBI group only $(n=8)$, CAPS scores were significantly reduced over time, $F(2,12)=16.6, p<.001$, pretreatment $(M=82.4, S D=11.7)$, posttreatment $(M=45.5, S D$ $=32.5$ ), pre- to posttreatment $d=1.51$. Further, the interaction 
Table 1

Hierarchical Linear Model of PTSD Checklist

\begin{tabular}{|c|c|c|c|c|}
\hline Effect & $\beta$ & $S E$ or variance component & $95 \% \mathrm{CI}$ & $t$ or $\chi^{2}$ \\
\hline \multicolumn{5}{|l|}{ Fixed Effect } \\
\hline Intercept & 54.42 & 1.90 & {$[50.70,58.14]$} & $28.63^{*}$ \\
\hline Weeks in treatment & -1.15 & 0.11 & {$[-1.37,-0.93]$} & $-10.24^{*}$ \\
\hline \multicolumn{5}{|l|}{ Random Effect } \\
\hline Intercept $\left(\mathrm{u}_{0}\right)$ & & 165.45 & {$[140.26,190.64]$} & $563.85^{*}$ \\
\hline Level 1 (r) & & 60.71 & {$[45.43,75.99]$} & \\
\hline
\end{tabular}

Note. $n=50, d f$ within $=233, d f$ between $=50 . \mathrm{PTSD}=$ posttraumatic stress disorder $\mathrm{CI}=$ confidence interval.

$* p<.001$.

between treatment group and time was also significant, $F(2,12)$ $=5.4, p=.02$. Follow-up planned $t$ tests between treatment groups at each time point revealed no difference at pretreatment (PE, $M=82.8, S D=15.3$; PCT, $M=82.0, S D=9.3, p=.70)$, no difference at midtreatment (PE, $M=55.8, S D=20.4$; PCT, $M=75.0, S D=23.2, p=.15)$, and approaching significance at posttreatment $(p=.06, \mathrm{PE}, M=24.8, S D=23.8$, PE preto posttreatment $d=2.9$; PCT, $M=66.3$, $S D=27.3$, PCT preto posttreatment $d=0.8$ ).

\section{Discussion}

In this study, we investigated the utility of PE for individuals with and without a history of TBI in a sample from a VHA PTSD clinic and in a small sample from a randomized controlled trial of PE versus PCT. As hypothesized, PE was highly efficacious for individuals with PTSD, and there was no evidence to suggest that the presence of TBI impacted the efficacy of PE. This is the first investigation to demonstrate a lack of difference in treatment trajectories from a direct comparison of PTSD plus TBI to PTSD alone.

Our findings replicate and extend previous reports that $\mathrm{PE}$ is effective for veterans with PTSD. Previous studies have demonstrated the efficacy of PE with multiera veterans (Rauch et al., 2009; Tuerk et al., 2011), female veterans (Schnurr et al., 2007), older veterans (Thorp, Stein, Jeste, Patterson, \& Wetherell, 2012), and via telehealth (Tuerk, Yoder, Ruggiero, Gros, \& Acierno, 2010). In our studies, PE demonstrated utility in a randomized controlled trial comparing PE versus PCT (see Rauch et al., 2013), as well as in a normative clinical sample of mixed traumas presenting to a VHA PTSD clinic. The effect size for PE was large (normative clinical sample, $d=1.13$; among TBI group in RCT, $d=2.9$ ). These effect sizes are similar to prior studies from our group (Rauch et al., 2009) and others (Schnurr et al., 2007). These data provide additional support for PE's role as a first-line treatment for PTSD in veteran populations, in accordance with the U.S. Department of Veterans Affairs \& Department of Defense (VA/DoD) Clinical Practice Guideline (VA/DoD, 2010).
Our findings provide preliminary evidence that mTBI status, which encompasses a broad range of severity of injuries, does not contraindicate PE. Similarly, Wolf and colleagues (2012) demonstrated a $45 \%$ post-PE reduction in PTSD symptoms in 10 veterans with PTSD plus mTBI. Our studies build on this finding in a number of ways. First, we establish that PE was helpful in a clinical sample of individuals both with and without a history of TBI. Second, HLM allowed us to examine change with both outcomes and slope of outcomes (i.e., trajectories of change) to provide additional evidence that individuals with PTSD plus TBI respond favorably to PE. Finally, our RCT sample of PTSD plus TBI demonstrated a trend toward lower posttreatment scores after PE than after PCT.

Some providers have voiced concerns that individuals with a history of mTBI are more likely to experience cognitive impairment and thus will not benefit from trauma-focused treatment (Bryant \& Hopwood, 2006; Elder et al., 2010; Otis et al., 2011). Our data challenge these concerns. In addition, the finding that $\mathrm{PE}$ is useful for individuals with a history of mTBI is consistent with PE's theorized mechanism of action. According to emotion processing theory (Rauch \& Foa, 2006), successful treatment requires modification of a fear structure through (a) activation of the fear structure, and (b) incorporation of incompatible corrective information into the fear structure (Foa \& Kozak, 1986). This is achieved through repeated exposure to situations and memories that are feared, but objectively safe, to facilitate habituation and extinction. Because these processes depend heavily on limbic and medial prefrontal circuits that are conserved across species (Quirk \& Mueller, 2008), there is little reason to believe that minor impacts on the brain would preclude them. Indeed, PE's emphasis on behavioral tasks makes it amenable to populations with varying levels of cognitive functioning, including children (Gilboa-Schechtman et al., 2010) and older adults (Thorp et al., 2012). Of note, the most commonly reported sequelae of mTBI-headache, dizziness, irritability, and memory problems (Terrio et al., 2009) — are equally likely to be present in individuals with PTSD but without mTBI (Brenner et al., 2010). Thus, it is unlikely that these physical and cognitive symptoms might serve as a basis for producing differential efficacy of PE in the PTSD with and without mTBI 
groups. In summary, PE may be well suited to those with a history of mTBI due to its simplicity, flexibility, and individualized nature (Rauch et al., 2012).

There are several limitations to this study. Our sample size was small, thus our results are not powered to address noninferiority questions regarding detecting medium to small PE-treatment differences that might exist between those with and without a history of TBI. We did not assess current postconcussive symptoms, only a history of TBI. Therefore, our study addresses historical TBI status and not necessarily PPCS. Some studies suggest PPCS is the mediating factor between TBI and PTSD (Morissette et al., 2011). Thus, it is possible that treating PPCS directly (through cognitive treatment, speech therapy, occupational therapy, or physical therapy) could assist in reduction of PTSD symptoms. However, mounting evidence suggests that PTSD prolongs and exacerbates PPCS (Hoge et al., 2008; Polusny et al., 2011); therefore, we believe that addressing PTSD symptoms directly in individuals with PPCS is warranted. To delineate this issue, future studies should distinguish between individuals with a history of mTBI who are currently experiencing PPCS versus individuals with a history of mTBI with no current PPCS. Our findings suggest that a history of mTBI does not preclude PE. A related issue is that our classification of TBI relied on chart review. Although all OEF/OIF/OND veterans were assessed for TBI via VHA standard TBI screening and received follow-up formal TBI consultation at dedicated TBI clinics, this stringent assessment could not be performed for Vietnam era and Persian Gulf veterans. Therefore, we relied on other means (service connection for TBI-related conditions; CPRS problem list) for identifying TBI among older veterans. This may have slightly impacted the precision of TBI diagnosis among older veterans. Additionally, level of cognitive function was not assessed in either study; as such, we cannot comment on cognitive function in our groups. However, the large treatment effect sizes in individuals with and without a history of TBI indicate that differences in cognitive functioning, if present, did not significantly impact treatment.

In conclusion, our study examined the impact of $\mathrm{PE}$ for individuals with and without a history of TBI, and found that the presence of TBI did not contraindicate PE. Because PTSD drives many psychosocial limitations in veterans with PTSD and mTBI, effective PTSD treatment for this population is critical for clinical care. The current findings suggest that as long as standard appropriate safety procedures are in place, treatment providers should continue to offer PE for PTSD to veterans with a history of mTBI, and to keep expectations for clinical improvements high.

\section{References}

American Congress of Rehabilitation Medicine. (1993). Definition of mild traumatic brain injury. Developed by the Mild Traumatic Brain Injury Committee of the Head Injury Interdisciplinary Special Interest Group of the American Congress of Rehabilitation Medicine. Journal of Head Trauma Rehabilitation, 8, 86-87.
American Psychiatric Association. (2000). Diagnostic and statistical manual of mental disorders (4th ed., text rev.). Washington, DC: Author.

Belanger, H. G., Curtiss, G., Demery, J. A., Lebowitz, B. K., \& Vanderploeg, R. D. (2005). Factors moderating neuropsychological outcomes following mild traumatic brain injury: A meta-analysis. Journal of the International Neuropsychological Society, 11, 215-227. doi:10.1017/S1355617705050277

Bisson, J. I., Ehlers, A., Matthews, R., Pilling, S., Richards, D., \& Turner, S. (2007). Psychological treatments for chronic post-traumatic stress disorder. Systematic review and meta-analysis. British Journal of Psychiatry, 190, 97-104. doi:10.1192/bjp.bp.106.021402

Blake, D. D., Weathers, F. W., Nagy, L. M., \& Kaloupek, D. G. (1995). The development of a Clinician-Administered PTSD Scale. Journal of Traumatic Stress, 8, 75-90. doi: 10.1002/jts.2490080106

Blanchard, E. B., Jones-Alexander, J., Buckley, T. C., \& Forneris, C. A. (1996). Psychometric properties of the PTSD Checklist (PCL). Behaviour Research and Therapy, 34, 669-673. doi: 10.1016/0005-7967(96)00033-2

Brenner, L. A., Ivins, B. J., Schwab, K., Warden, D., Nelson, L. A., Jaffee, M., \& Terrio, H. (2010). Traumatic brain injury, posttraumatic stress disorder, and postconcussive symptom reporting among troops returning from Iraq. Journal of Head Trauma Rehabilitation, 25, 307-312. doi:10.1097/HTR.0b013e3181cada03

Bryant, R. A. (2001). Posttraumatic stress disorder and traumatic brain injury: Can they co-exist? Clinical Psychology Review, 21, 931-948.

Bryant, R. A., \& Hopwood, S. (2006). Commentary on "trauma to the psyche and soma." Cognitive and Behavioral Practice, 13, 17-23.

Carlson, K., Kehle, S., Meis, L., Greer, N., MacDonald, R., Rutks, I., \& Wilt, T. J. (2009). The assessment and treatment of individuals with history of traumatic brain injury and post-traumatic stress disorder: A systematic review of the evidence. Washington, DC: U.S. Department of Veterans Affairs.

Chard, K. M., Schumm, J. A., McIlvain, S. M., Bailey, G. W., \& Parkinson, R. B. (2011). Exploring the efficacy of a residential treatment program incorporating cognitive processing therapy-cognitive for veterans with PTSD and traumatic brain injury. Journal of Traumatic Stress, 24, 347-351. doi: $10.1002 /$ jts. 20644

Defense Manpower Data Center. (2009). Contingency tracking system. Retrieved from http://www.dmdc.osd.mil/appj/dwp/getLinks.do? category $=$ dod $\&$ subCatda-taInq $\&$ tab $=3 \& c 1 O n=$ dod

Elder, G. A., Mitsis, E. M., Ahlers, S. T., \& Cristian, A. (2010). Blast-induced mild traumatic brain injury. Psychiatric Clinics of North America, 33, 757781. doi:10.1016/j.psc.2010.08.001

Foa, E. B., Hembree, E., \& Rothbaum, B. O. (2007). Prolonged exposure therapy for PTSD: Therapist guide. New York, NY: Oxford University Press.

Foa, E. B., \& Kozak, M. J. (1986). Emotional processing of fear: Exposure to corrective information. Psychological Bulletin, 99, 20-35. doi: 10.1037/0033-2909.99.1.20

Gilboa-Schechtman, E., Foa, E. B., Shafran, N., Aderka, I. M., Powers, M. B., Rachamim, L., . . A Apter, A. (2010). Prolonged exposure versus dynamic therapy for adolescent PTSD: A pilot randomized controlled trial. Journal of the American Academy of Child and Adolescent Psychiatry, 49, 1034-1042. doi:10.1016/j.jaac.2010.07.014

Hoge, C. W., Goldberg, H. M., \& Castro, C. A. (2009). Care of war veterans with mild traumatic brain injury-flawed perspectives. New England Journal of Medicine, 360, 1588-1591. doi:10.1056/NEJMp0810606

Hoge, C. W., McGurk, D., Thomas, J. L., Cox, A. L., Engel, C. C., \& Castro, C. A. (2008). Mild traumatic brain injury in U.S. soldiers 
returning from Iraq. New England Journal of Medicine, 358, 453-463. doi:10.1056/NEJMoa072972

Independent Review Group. (2007). Rebuilding the trust: Report on rehabilitative care and administrative processes at Walter Reed Army Medical Center and National Naval Medical Center. Retrieved from http://www.ha.osd.mil/dhb/recommendations/2007/IRG-Report-Final.pdf

Joseph, S., \& Masterson, J. (1999). Posttraumatic stress disorder and traumatic brain injury: Are they mutually exclusive? Journal of Traumatic Stress, 12, 437-453. doi:10.1023/A:1024762919372

Monson, C. M., Gradus, J. L., Young-Xu, Y., Schnurr, P. P., Price, J. L., \& Schumm, J. A. (2008). Change in posttraumatic stress disorder symptoms: Do clinicians and patients agree? Psychological Assessment, 20, 131-138. doi:10.1037/1040-3590.20.2.131

Morissette, S. B., Woodward, M., Kimbrel, N. A., Meyer, E. C., Kruse, M. I., Dolan, S., \& Gulliver, S. B. (2011). Deployment-related TBI, persistent postconcussive symptoms, PTSD, and depression in OEF/OIF veterans. Rehabilitation Psychology, 56, 340-350. doi:10.1037/a0025462

Otis, J. D., McGlinchey, R., Vasterling, J. J., \& Kerns, R. D. (2011). Complicating factors associated with mild traumatic brain injury: Impact on pain and posttraumatic stress disorder treatment. Journal of Clinical Psychology in Medical Settings, 18, 145-154. doi:10.1007/s10880-011-9239-2

Owens, B. D., Kragh, J. F., Jr., Wenke, J. C., Macaitis, J., Wade, C. E., \& Holcomb, J. B. (2008). Combat wounds in operation Iraqi Freedom and operation Enduring Freedom. Journal of Trauma, 64, 295-299. doi:10.1097/TA.0b013e318163b875

Polusny, M. A., Kehle, S. M., Nelson, N. W., Erbes, C. R., Arbisi, P. A., \& Thuras, P. (2011). Longitudinal effects of mild traumatic brain injury and posttraumatic stress disorder comorbidity on postdeployment outcomes in national guard soldiers deployed to Iraq. Archives of General Psychiatry, 68, 79-89. doi:10.1001/archgenpsychiatry.2010.172

Powers, M. B., Halpern, J. M., Ferenschak, M. P., Gillihan, S. J., \& Foa, E. B. (2010). A meta-analytic review of prolonged exposure for posttraumatic stress disorder. Clinical Psychology Review, 30, 635-641. doi:10.1016/j.cpr.2010.04.007

Quirk, G. J., \& Mueller, D. (2008). Neural mechanisms of extinction learning and retrieval. Neuropsychopharmacology, 33, 56-72. doi:10.1038/sj.npp.1301555

Rauch, S. A. M., Defever, E., Favorite, T., Duroe, A., Garrity, C., Martis, B., \& Liberzon, I. (2009). Prolonged exposure for PTSD in a Veterans Health Administration PTSD clinic. Journal of Traumatic Stress, 22, 60-64. doi: $10.1002 /$ jts. 20380

Rauch, S. A. M., Eftekhari, A., \& Ruzek, J. I. (2012). Exposure therapy: A gold standard for PTSD treatment. Journal of Rehabilitation Research and Development, 49, 679-687. doi:10.1682/JRRD.2011.08.0152

Rauch, S. A. M., \& Foa, E. B. (2006). Emotional processing theory (EPT) and exposure therapy for PTSD. Journal of Contemporary Psychotherapy, $36(2), 61-65$

Rauch, S. A. M., King, A. P., Tuerk, P. W., Smith, E., Giardino, N. D., Rothbaum, B. O., .. . Liberzon, I. (2013). Changes in symptoms, cognitions, and HPA axis function with treatment of OEF/OIF veterans with chronic PTSD. Manuscript submitted for publication

Raudenbush, S. W., \& Bryk, A. S. (2002). Hierarchical linear models: Applications and data analysis methods (2nd ed.). Newbury Park, CA: Sage.

Raudenbush, S. W., Bryk, A. S., Cheong, Y. F., \& Congdon, R. T. J. (2004) HLM 6: Hierarchical linear and nonlinear modeling. Lincolnwood, IL: Scientific Software International.
Schneiderman, A. I., Braver, E. R., \& Kang, H. K. (2008). Understanding sequelae of injury mechanisms and mild traumatic brain injury incurred during the conflicts in Iraq and Afghanistan: Persistent postconcussive symptoms and posttraumatic stress disorder. American Journal of Epidemiology, 167, 1446-1452. doi:10.1093/aje/kwn068

Schnurr, P. P., Friedman, M. J., Engel, C. C., Foa, E. B., Shea, M. T., Chow, B. K., . . Bernardy, N. (2007). Cognitive behavioral therapy for posttraumatic stress disorder in women: A randomized controlled trial. Journal of the American Medical Association, 297, 820-830. doi:10.1001/jama.297.8.820

Sheehan, D. V., Lecrubier, Y., Sheehan, K. H., Amorim, P., Janavs, J., Weiller, E., ... Dunbar, G. C. (1998). The Mini-International Neuropsychiatric Interview (M.I.N.I.): The development and validation of a structured diagnostic psychiatric interview for DSM-IV and ICD-10. Journal of Clinical Psychiatry, 59(Suppl. 20), 22-57.

Snijders, T., \& Bosker, R. (1994). Modeling variance in two-level models. Sociological Methods and Research, 22, 342-363.

Tanielian, T. L., \& Rand Corporation. (2008). Invisible wounds of war: Summary and recommendations for addressing psychological and cognitive injuries. Santa Monica, CA: RAND Corp.

Terrio, H., Brenner, L. A., Ivins, B. J., Cho, J. M., Helmick, K., Schwab, K., . . W Warden, D. (2009). Traumatic brain injury screening: Preliminary findings in a US Army Brigade Combat Team. Journal of Head Trauma Rehabilitation, 24, 14-23. doi:10.1097/HTR.0b013e31819581d8

Thorp, S. R., Stein, M. B., Jeste, D. V., Patterson, T. L., \& Wetherell, J. L. (2012). Prolonged exposure therapy for older veterans with posttraumatic stress disorder: A pilot study. American Journal of Geriatric Psychiatry, 20, 276-280. doi:10.1097/JGP.0b013e3182435ee9

Tuerk, P. W., Yoder, M., Grubaugh, A., Myrick, H., Hamner, M., \& Acierno, R. (2011). Prolonged exposure therapy for combat-related posttraumatic stress disorder: An examination of treatment effectiveness for veterans of the wars in Afghanistan and Iraq. Journal of Anxiety Disorders, 25, 397-403. doi:10.1016/j.janxdis.2010.11.002

Tuerk, P. W., Yoder, M., Ruggiero, K. J., Gros, D. F., \& Acierno, R. (2010). A pilot study of prolonged exposure therapy for posttraumatic stress disorder delivered via telehealth technology. Journal of Traumatic Stress, 23, 116123. doi: $10.1002 /$ jts. 20494

U.S. Department of Defense. (1998). Joint tactics, techniques and procedures for antiterrorism (Vol. 3-07.2). Washington, DC: Author.

U.S. Department of Veterans Affairs \& Department of Defense (VA/DoD). (2010). VA/DoD clinical practice guideline for the management of posttraumatic stress. Washington, DC: Author.

Verfaellie, M., Amick, M. M., \& Vasterling, J. J. (2012). Effects of traumatic brain injury-associated neurocognitive alterations on posttraumatic stress disorder. In J. J. Vasterling, R. A. Bryant, \& T. M. Keane (Eds.), PTSD and mild traumatic brain injury (pp. 83-102). New York, NY: Guilford Press.

Walter, K. H., Kiefer, S. L., \& Chard, K. M. (2012). Relationship between posttraumatic stress disorder and postconcussive symptom improvement after completion of a posttraumatic stress disorder/traumatic brain injury residential treatment program. Rehabilitation Psychology, 57, 13-17. doi: $10.1037 / \mathrm{a} 0026254$

Weathers, F. W., Huska, J. A., \& Keane, T. M. (1991). PCL-S for DSM-IV. Boston, MA: National Center for PTSD, Behavioral Science Division.

Wolf, G. K., Strom, T. Q., Kehle, S. M., \& Eftekhari, A. (2012). A preliminary examination of prolonged exposure therapy with Iraq and Afghanistan veterans with a diagnosis of posttraumatic stress disorder and mild to moderate traumatic brain injury. Journal of Head Trauma Rehabilitation, 27, 26-32. doi:10.1097/HTR.0b013e31823cd01f 